\title{
NoObesity Apps - from approach to finished app
}

\author{
Authors: Denyse King ${ }^{1}$, Em Rahman ${ }^{2}$, Alison Potter ${ }^{3}$ and Edwin van Teijlingen ${ }^{4}$ \\ ${ }^{1}$ Bournemouth University East Branch Campus, Midwifery Education, $4^{\text {th }}$ Floor \\ SMCHC, Milton Road, Portsmouth, PO3 6AD, England, UK \\ dking@bournemouth.ac.uk \\ ${ }^{2}$ Southern House, Otterbourne, Winchester, Hants, SO21 2RU, England, UK \\ ${ }^{3}$ Southern House, Otterbourne, Winchester, Hants, SO21 2RU, England, UK \\ ${ }^{4}$ Bournemouth University, Bournemouth House, 19 Christchurch Road, \\ Bournemouth, BU1 3LH, England, UK
}

\section{Please quote this is:}

\begin{abstract}
King, D., Rahman, E., Potter, A., van Teijlingen, E. (2019) NoObesity apps - From approach to finished app. In: Arai K., Bhatia R., Kapoor S. (eds) Proceedings of the Future Technologies Conference (FTC) 2018. FTC 2018. Advances in Intelligent Systems \& Computing Vol. 881: 1145-1157, Springer, Cham 10.1007/978-3-03002683-7_84
\end{abstract}

\begin{abstract}
Obesity is still a growing public health problem in the UK and many healthcare workers find it challenging to have a discussion with service users about this sensitive topic. They also feel they are not competent to provide the relevant heath advice and are seeking easily accessible, evidence-based, mobile health learning (mHealth). mHealth applications (apps) such as the Professional NoObesity and Family NoObesity (due for release late 2018), have been designed to: support families with making sustainable positive behaviour changes to their health and well-being, ease pressure on practitioners' overweight and obesity care related workloads, as well as to support the education of professionals, students and service users. This paper describes the process of designing the apps from the inception of the idea, through the stages of research, app builds and testing. The processes of collaborative working to design and develop the apps to meet the needs of both service users and health professionals will also be reflected upon. Childhood obesity is an complex problem and whilst it is recognised that the NoObesity apps cannot singlehandedly resolve this health crisis, it is proposed that they can support families to identify and reduce the barriers that prevent them from living healthier, happier lives.
\end{abstract}

Key words: Mobile [mHealth] learning, eLearning, collaboration 


\section{Introduction}

Public Health England's (PHE) National Childhood Measurement Programme (NCMP) shows a downward trend for excess weight, overweight and obesity in boys aged 4-5 (=reception) over the past decade (Fig 1), but a year-on-year upward trend for obesity and excess weight for boys and girls aged 10-11 (=year 6) [1].

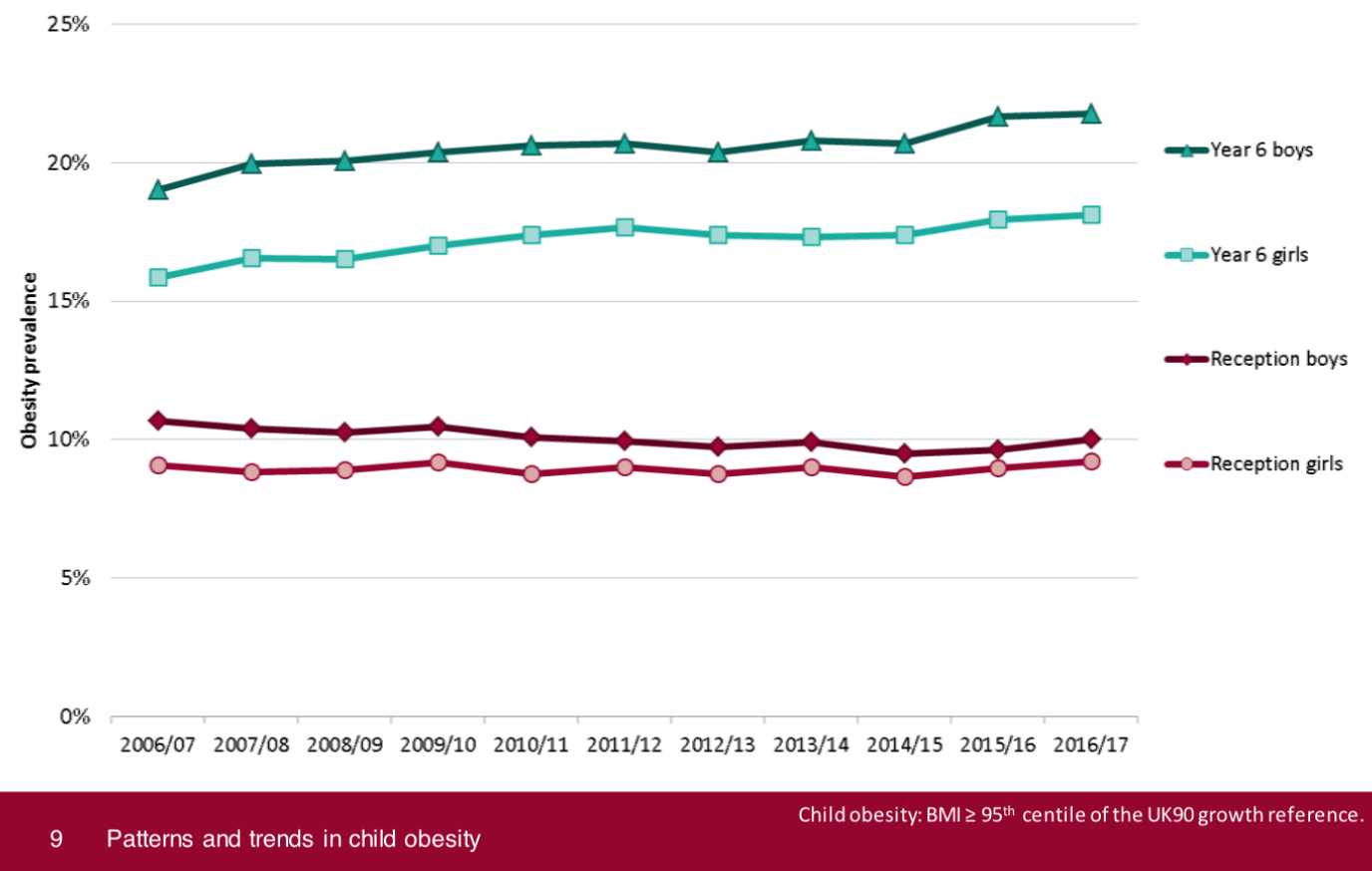

Fig 1 Prevalence of obesity by age and sex. [2]

The health secretary announced at the 2015 PHE conference that one of the government's key priorities is reducing childhood obesity through adult education. However, research by King [3] demonstrated that health professionals felt that they already made weight, overweight or obesity part of their routine healthcare consultations. Therefore, it could be argued that the solution may not be as simple as 'educating the adults', and perhaps professionals need to refresh their learning delivery techniques or that the 
method for delivering such education should be modernised. A systemic review by Mosa et al. [4] highlighted the importance of mobile healthcare (mHealth), which is already being used successfully to enhance current healthcare provision [5]. Research conducted by King [3] with service users and health professionals highlighted the potential for mHealth in relation to use for families with overweight or obese children and their health professionals. Cugelman [6] suggested that gamification principles are closely related to principles that have been proven to work in relation to behaviour change such as challenges and effective motivation to maximise potential. Whilst it is acknowledged that gaming itself may not seem appropriate for healthcare education, its principles may be effective in relation to facilitating mHealth behaviour change.

\section{Background}

One of the UK government's key priorities is reducing childhood obesity through adult education. However, discussing obesity with patients can be difficult for healthcare workers and many do not feel confident enough to initiate such conversations and complete them with competence. The Maternal and Childhood Obesity (MaCO) package is Bournemouth University's standalone distance e-Learning, designed to support healthcare workers in increasing their knowledge and confidence needed to have conversations with services users who are overweight or obese, or whose children are [7]. MaCO can be studied anywhere as long as the learner has an internet connection. 
Learner' feedback has demonstrated that MaCO has the long-term potential to improve maternal and childhood health outcomes through the provision of effective advice and guidance; helping service users to make suitable lifestyle choices and encouraging behavioural motivations. However, it does not readily support those who need ad-hoc or offline learning and support whilst working clinically. Furthermore it does not offer service users the opportunity to learn how to make behavioural modification to their own health and wellbeing beyond the consultation with their named healthcare worker. Research undertaken with healthcare workers and families confirmed the need for mHealth content individually targeted to help achieve the best outcomes for both. Bennett et al. [8] argued almost ten years ago that children are digital natives, and that adults are rapidly becoming digital 'immigrants' who will turn to mobile technology when seeking answers [9]. Topol [10] noted that healthcare is moving towards digital medicalisation thereby allowing healthcare to be increasingly delivered by wireless technology which in turn will empower the patient to not only take more responsibility for their healthcare and work in partnership with their provider of health improvements. Dennison et al. [11] concurred, stating that smartphones are have the potential to deliver health interventions but cautioned that there were limitations such as providing accurate and timely information with minimal effort required from the user, which could restrict length of engagement. Interestingly, the National Institute for Health Care and Excellence [(NICE) 12] also made a recommendation for consideration of the use of mobile technology to support individual behaviour change. A study conducted by Morrison et al. [13] concluded that smart phones were linked to increasing 
engagement with health-related goals. The above combination of factors gave rise to a proposal being put forward to Health Education England to develop learning content that could be used with mobile learning technology to facilitate positive change in relation to obesogenic behaviours.

\section{Methodology and methods}

The research was undertaken using a constructionist approach. This qualitative study identifies people's perceptions and understanding of their health care experience. Phenomenology is situated in the interpretive approach that allows analysis of experiences via descriptions and can thereby give voice to that which would not normally be made explicit [14]. This methodology helped to identify ways to improve obesity health services from the perspectives of service users and the health professionals who currently provide care to those who are overweight or obese. Ethics approval for the project was granted by Bournemouth University and participants were sourced via healthcare workers' word of mouth, and social media using hashtags such as \#yourhealthcare, \#healthcareresearch, \#healthcareteam, \#healthcareprofessional, and \#pleaseRT on social media platforms including Twitter, Facebook, Instagram and blogs. Volunteering participants were given further information about the research and an opportunity to give consent prior to proceeding with the research which was conducted in 2016. Given the UK's key priority to reduce obesity in children through education of their parents, this research sought participants who were parents with experience of healthcare related to their children's weight and health professionals responsible for providing care to overweight or obese children. Healthcare 
workers and service users were engaged effectively through surveys and online focus groups [15]; 17 service users, two members of the general public and 32 health professionals participated in the preliminary research which consisted of open, closed and Likert-scale questions (Appendices A \& B) and helped to inform the design and first draft of the content of the apps.

\subsection{Service user survey, focus group and sampling}

The service user focus groups were all undertaken online using the FocusGroupit platform where 13 participants responded to questions set by the interviewer and also discussed questions with each other. The service user survey was undertaken using the Survey Monkey platform and six participants chose this method to respond to the questions.

The service user survey and online focus group topic questions were designed to determine their pre-existing perceptions of overweight and obesity, their experience to date with healthcare for the same, what barriers there were that prevented them from making positive changes to their health and wellbeing, and what tools they would find useful to support them identifying and making positive behaviour changes.

\subsection{Health professional survey, focus group and sampling}

The online focus group included 18 health professionals and 14 contributed to a survey using the same platforms as described above. They were invited to respond to a range of questions targeted at determining what mHealth functionality would be best suited to improve their skills and confidence related to supporting families with overweight or obese children to identify and 
make positive, realistic and sustainable lifestyle changes. The answer transcripts were analysed to identify themes within their responses. This method of data collection captured insights which helped to identify the challenges and issues experienced when trying to prevent / manage overweight and obesity.

Once the content draft was written, and approved by the steering group, service users and general public were asked to review the apps' content. They provided requested feedback on whether the content was easy to read and compelling, suitable and likely to help their family to make healthier choices, what could be improved and what they liked about the content, Health professionals were also asked to review the content and asked similar questions including whether they felt the content had currency for their area of work and practice and would help them to support families to make healthier choices. Their feedback was applied as appropriate and then a prototype of the core functionality in the Family app and the Health Professional app were created. These prototypes consisted of visual designs of key screens which testers could click through, 13 people participated in the Family app user acceptability testing and 13 participated in the user acceptability testing of the health professional version of the app after which further modifications were made before moving to production. In total the app development company completed 23 builds before the apps were ready for the final user acceptability testing (UAT).

\section{Research findings}


Key themes related to service users and providers in relation to support around reducing obesity were:

- 15 out of 19 service users responding felt that children don't always react well to parents telling them what to do:

"I didn't tell her as I didn't want her to have self esteem issues and be body conscious." Respondent 11

- 12 out of 19 service users wanted to be taught by professionals that have experience of teaching parents

"Look at the cause, before dictating a solution." Respondent 5

- 11 out of 19 service users wanted to feel they are being given individualised advice

"Be sympathetic and come up with realistic responses not text book."

\section{Respondent 9}

- 14 out of 19 service users didn't want to have to spend money and time they didn't have on healthier lifestyles:

"We want to make our lives as easy and habitual as possible, so finding more time in our already busy days for meal planning, shopping, food preparation, eating and clearing up is being neglected more and more as processed 'convenience foods' are the preferred foods of choice." Respondent 3

- Two-thirds of professionals wanted the app content to be evidence based in order for it to offer full value "Need current and up-to-date training and knowledge." Respondent 5 
- 22 out of 32 professionals feel they do make weight part of their routine health conversation with service users but often do not feel confident having conversations with services users about overweight and obesity.

"People get very defensive though and take it as a personal insult!"

Respondent 4

"It is a fine balance between being seen as knowledgeable and judging."

\section{Respondent 13}

From the above key themes, it was clear that the apps needed to:

- Empower -to recognise the experiences people bring and therefore the tools had to be empowering in supporting families to address obesity.

- Offer parenting tips - to address challenges with encouraging positive health behaviours with children.

- Be responsive to barriers - put up by parent/carers who are being supported by healthcare workers and help them to identify individualised solutions.

- Acknowledge - that sustained behaviour change takes time and that support for overcoming barriers is vital.

- Provide easy and simple ideas - to support parents/carers and professionals to identify quick ways to support healthier eating and increase activity.

- Highlight - that portion size is important in addition to eating healthily and exercising. 
Our findings mirror Lupton's [16] advisory caution that app developers need to ensure there is a balance between what is promised from use of the apps with what can realistically be expected from the average app users as well as mitigating the risk of the app content appearing to be coercive. It was also clear that the development of this project pivoted on putting service users at the centre of the behaviour change process. This ties in with Rollnick's [17] suggestion that there is a need to move towards a partnership model, with service users being supported to assume ownership of their health decisions by the healthcare workers. The importance of this is supported by the eight dimensions of humanisation of healthcare which puts the individual at the cente of care [18].

\section{Collaborative processes}

The NoObesity apps were designed and developed by a project collaborative formed of health professionals, academics, technical experts from the app development company, behaviour specialists, teachers, childhood obesity specialists, and public health specialists. Collaborative working facilitated the design of apps and content was written to provide individual user specific evidence-based information, guidance and support to families and health professionals. A design day was held to identify ways in which the research findings could be addressed throughout the apps. The key themes from the research had been developed into personas by the app content author which were then used in the design process to identify and agree the app sections. The personas helped to keep design day participants focused on the key aim of the apps; to support service users and professionals with challenging and 
working towards changing overweight and obesogenic behaviours. The project collaborative worked together to develop solutions to the issues and challenges identified by the research. These proposed solutions were then tested further with service providers and users which informed the final structure and functions to be made available in the finished apps.

\subsection{Project Steering Group}

Following the app design day, topic experts were identified to complete the formation of the steering group which included members from around the UK. This group met quarterly to provide the governance, steer and topic expertise to the overall development of this project. The apps' content was written using a person-centred approach where service users were made central to the care offered by the apps through application of Wessex Making Every Contact Count (MECC) techniques [19]. The steering group were asked to review the content of the apps after it was written by the first author. This led to some challenging discussions in which many professional viewpoints and discipline specific agendas were proposed or contested. Due to all members of the steering group understandably wishing for the apps to provide the optimum focus related to their specific discipline, and the need for balance in the overall advice given, there was some instances of protracted discussion in face to face meetings and subsequent virtual meetings until majority agreement could be reached.

\section{Discussion}


The apps' content was written to contribute to the childhood obesity agenda through training and education of the healthcare workforce with a modern edge added by offering the content as an app to support mHealth learning for both service users and service providers as well as healthcare students. The apps were named NoObesity Professional and NoObesity Families by children who took part in a primary and secondary school competition across England. This method of naming the apps was chosen to make them more appealing to service users who would be within the same age demographic when the apps were recommended to them as part of their weight management consultation.

The NoObesity Professional app is a set of tools that can be used with families as part of a consultation visit to increase the confidence, knowledge and skills of the parents/carers of children who are obese or overweight. It also includes learning content - in the form of case studies - to help familiarize healthcare workers and healthcare students with holding conversations about healthy weight and lifestyle choices. Healthcare workers can see the goals, barriers, strategies, progress, points and awards of linked families, making them better able to provide tailored advice to the families, to help them achieve their goals. This is based on research findings that standard generic health advice simply doesn't work for most families.

"No one size fits all and we are all different and have different needs" Respondent 2

"When you don't understand the measurements being used to assess your child, and how changeable the whole thing is, if your child doesn't fit into what is considered normal then the feedback can be worrying." Respondent 1 
"They were clear with their information, but it was inconsistent advice and didn't seem to take my opinion into account." Respondent 12

Both NoObesity apps include the Wessex MECC approach which is designed to support service users with identifying their own solutions for change [19]. The NoObesity Professional app also contains advice on how to best support service users in the context of the family as a whole, how to handle common objections from parents and children, progressive scenarios for immersive learning, games, learning challenges and useful links. In addition, and intended as a companion to NoObesity Professional app, the NoObesity Families app content can be used by families when they are on their own without the support of their healthcare workers. After consultation with a healthcare worker, families set health goals, identify potential barriers and strategies to overcome them, record their progress towards their goals, earning points and awards as they go. Families are encouraged to link accounts to healthcare worker accounts. The NoObesity apps' content also include parenting tips, games for the children and parents to use for learning about portion sizes, energy expenditure, how to change recipes to healthier versions and useful links to free family outings and local exercise opportunities. Kayyali et al. [20] intimate that counselling and health information can be included within app games to increase appeal and subsequent use. This further supports the research conducted by the Royal Society of Public Health [21] who published a childhood obesity strategy in which they stated that over $85 \%$ of children wanted to learn more about nutrition, and to receive increased promotion of age appropriate healthier physical activities, amongst other advice. Whilst it can be seen that games 
within the apps as a method for delivering information will certainly appeal to the younger NoObesity app users, Knight et al. [22] highlight the need for mHealth apps to be evidence based in order to be useful for improving health. Those who contributed to the health professionals' aspect of the research for the NoObesity apps project $(n=19)$ concurred with this view and the other 23 chose not to contribute their opinion about what professional content they would find helpful. Based on the information from those who contributed, it was clear that the content in the professionals' app should encapsulate evidence-based information in order to engage the professional user, support them during consultations with service users, as well as reward the professionals for using the apps to increase their personal learning and encourage them to return to the app on a regular basis to further improve their knowledge and confidence in relation to supporting service users to make positive lifestyle changes.

Therefore, the aims of the apps are to achieve a change in service users' attitudes in relation to the importance of a healthy weight and lifestyle and to achieve a change in the service providers' attitude towards supporting clients with behavioural modification related to reducing weight and improving lifestyle. The ultimate intended outcome is that these apps encourage and support positive lifestyle changes to become autonomous at an individual client led level and that these are sustainable and transferrable from parent to child.

\section{Lessons learned and potential limitations}


The primary proposal had been to research, develop and write content for one app for health professionals to use for their own learning and to support them during consultations with overweight and obese service users. Research identified that there was a need for two targeted apps rather than the one that was initially proposed, in order to allow focus of information provision to be steered appropriately. This streamlined focus was for two main reasons: first, families with overweight or obese individuals are already aware their lifestyles are less than ideal. Families want to make changes, but feel stuck in a loop of unhealthy but comfortable routine family behaviours. Secondly, healthcare workers want to reduce their overweight / obesity workload. Information from stakeholders, and research with service users and healthcare workers, meant that the app content was written to straddle the divide between what families wanted from their healthcare professional with what was reasonable and realistic to expect from current health services care without increasing staffing levels or extending work days. Collaboration through involvement of the service users, health professionals, healthcare workers, students and academics throughout the design process, helped to keep the aims of the project focussed though this occasionally led to challenging conversations when trying to ensure the content reflected the key aspects of each expert's profession in a balanced or proportionally significant measure. The steering group was carefully chosen to ensure key disciplines related to overweight and obesity lifestyles and healthcare were covered whilst keeping the numbers of the steering group to an irreducible minimum. When the content was being reviewed there were disputes which occasionally could not be resolved easily and which necessitated the need for Chair's action or 
moderation from additional subject experts. However, this ultimately led to further robustness of the apps' content and it could be argued that this indicates that there was a need for these additional experts to be part of the steering group from the beginning of the project. These apps were designed based on service user and health professional feedback on what they felt would make an app to support healthy weight behaviours and other lifestyle changes. The research sample sizes were small, and the participants were motivated to take part in the research due to personal experience with receiving or providing overweight or obesity related healthcare advice. Further research needs to be done when the apps are released to ascertain families' level of and length of engagement with the app content, whether they choose to link to health professionals' NoObesity apps, and if so which ones they link to, as well as families' recorded progress towards their chosen behaviour change goals. Furthermore, use by professionals needs to be monitored to identity if they are using the apps on a regular basis, and whether this is for personal learning, as part of their consultation with the service usrr or a combination of the two as well as whether the professional continues to use the apps over a sustained period of time. Finally, changes to how organisations process personal data when the new EU general data protection regulation (GDPR) law came into effect in May 2018 has created considerable challenges in finding an organisation that could assure they met these new requirements in order to support the backend data for the apps. This consequently delayed the apps' release date for use by health professionals and service users and our future research. 


\section{Conclusion}

The project developed two standalone apps which can be paired to expand their use. The professionals' app delivers storyline-driven content to offer authentic practice within realistic scenarios, and the enquiry-based behavior change content of the families' app encourages and sustains the drive to selfmotivate on the part of the service users. When these apps are paired the families can communicate progress towards goals to their healthcare worker who can then prepare for subsequent consultations with the family in advance of their scheduled appointment. Future research to investigate the success of the app content in supporting and sustaining positive behaviour and lifestyle change begins after the apps have been released for free download in late 2018. 


\section{References}

1. Copley V. R.,Bray C.: National Child Measurement Programme: changes in children's body mass index in England between 2006 to 2007 and 2015 to 2016. London: Public Health England (2017).

2. Public Health England Patterns and trends in childhood obesity, A presentation of the latest data on child obesity PHE Publishing Ltd, London, PHE publications gateway number: 2017776 (2018)

3. King, D., unpublished transcripts from online focus groups conducted as preliminary research into what healthcare workers and service users want in relation to overweight and obesity healthcare (2016)

4. Mosa, A.S.M.; Yoo, I.; Sheets, L. A systematic review of healthcare applications for smartphones. BMC Medical Informatics and Decision Making (2012) 12:67 http://www.biomedcentral.com/1472-6947/12/67

5. Fors., U., Courteille, O.: Learner acceptance of using virtual patient encounters to train foreign healthcare professionals in Swedish, International Journal of Virtual \& Personal Learning Environments, 5, (3) 18-32, (2014).

6. Cugelman, B Gamification: what it is and why it matters to digital health behaviour change. JMIR serious games, 1, (1) 3 (2013)

7. King, D., Maternal and Childhood Obesity online standalone distance learning package for healthcare professionals and healthcare workers Bournemouth University, England (2009)

8. Bennett S., Maton K.A., Kervin, L.: 'The 'digital natives' debate: a critical review of the evidence', British Journal of Educational Technology, 39(5), 775-86 (2008)

9. King, D., unpublished formative doctoral essay on learning submitted to Bournemouth University, England (2018)

10. Topol, E., The Patient Will See You Now: The Future of Medicine is In Your Hands. ${ }^{\text {st }}$ edn. Basic Books: New York, NY. (2015)

11. Dennison, L., Morrison, L., Conway, G., Yardley, L.: Opportunities and Challenges for Smartphone Applications in Supporting Health Behavior Change: Qualitative Study. Eysenbach G, ed. Journal of Medical Internet Research. 2013;15(4):e86. doi:10.2196/jmir.2583.

12. NICE guideline $\mathrm{PH} 49$, recommendation no 7 https://www.nice.org.uk/guidance/ph49 (2014)

13. Morrisson, L.G., Hargood, C., Lin, S.X., Dennison, L., Joseph, J., Hughes, S., Michaelides, D.T., Johnston, M., Michie, S., Little, P., Smith, P.W., Wel, M.J., Yardley, L.: Understanding usage of a hybrid website and smartphone apps for weight management: a mixed method study Journal of Medical Internet Research 16(10), e201 (2014)

14. Donaleck, J.: Phenomenology as a Qualitative Research Method. Urologic Nursing, 24 (6), 516-517. (2004).

15. Stancanelli, J.: Conducting an Online Focus Group. The Qualitative Report, 15(3), 761-765. (2010) Retrieved from http://nsuworks.nova.edu/tgr/vol15/iss3/20 accessed Feb 18th 2017

16. Lupton, D.: I just want it to be done, done, done!' Food tracking apps, affects, and agential capacities Multimodal Technologies Interact. 2(2), 29 (2018) https://doi.org/10.3390/mti2020029 - 23 May 2018 
17. Rollnick, R Miller, W., Butler, C.: Motivational interviewing in Health Care: Helping Patients Change Behaviour (Applications of motivational interviewing) Guildford Press (2008)

18. Hemingway, A., Scammell, J., Heaslip, V.: Humanising nursing care: a theoretical model Nursing Times 108(40), 26-27 (2012)

https://www.nursingtimes.net/Journals/2012/09/28/s/u///021012-Humanisingnursing-care--a-theoretical-model-pdf

19. Lawrence, W., Cheminade, C., Em Rahman., Annemarie Hankinson.: MECC Training Manual, Health Education England (2018) www.wessexphnetwork.org/MECC

20. Kayyali, R., Peletidi A, Ismail, M, Hashim, Z, Bandeira, P., Bonnah, J.: Awareness and Use of mHealth Apps: A Study from England. Pharmacy,5(2),33 (2017).

21. Royal Society for Public Health The Child's Obesity Strategy: how our young people would solve the childhood obesity crisis, London (2017)

22. Knight, E., Stuckey, M.I., Prapavessis, H., Petrella, R.J.: Public Health Guidelines for Physical Activity: Is There an App for That? A Review of Android and Apple App Stores. Eysenbach, G., ed. JMIR mHealth and uHealth. 2015;3(2): e43. doi:10.2196/mhealth.4003. 


\section{Appendix A}

Research questions asked of service users (all included a space to expand on responses)

1. What are your views on childhood overweight and obesity? Please share any personal experience or anecdotes.

2. Have you, your child, or a child you know ever had healthcare advice related to them being overweight?

3. If you or your child were given advice about lifestyle to help reduce weight, did you find this a positive or negative experience?

4. If a healthcare worker gives you advice about yours or your child's weight, do you understand what they mean?

5. Do you feel you can ask questions when a healthcare worker gives you weight advice for you or your child?

6. Do you know what questions you want to ask when a healthcare worker gives you weight advice for you or your child?

7. How do you prefer to be given advice by healthcare workers about your or your child's weight?

8. What can healthcare workers do to help make the experience of being given weight advice more useful for you and your child?

9. Do you feel you are aware of the difference between child and adult portion sizes?

10. Please describe a typical breakfast, lunch and supper your child (or the child you wish to discuss) eats including how much of each food they have (for example, how many slices of bread used for sandwich, which and how many 
fruit and veg, types of meat or meat substitute, dairy, sweets, crisps, readymade or fast foods, drinks).

11. Do your children do any exercise?

12. Are you aware of any free exercise sessions in your local area, and if so can you name them?

13. What kind of lifestyle support or advice would you find useful?

14. Do you know of any apps / websites that support healthy lifestyles and does your family use them? 


\section{Appendix B - Research questions asked of health professionals (all}

included a space to expand on responses)

1. What setting do you work in?

2. What are your views on childhood obesity? Please share any personal experience or anecdotes.

3. Do you make infant / childhood weight part of your routine health conversation with parents / patients?

4. How do you support parents and children to modify challenging behaviour? What works well for you?

5. What are your concerns in relation to working with parent and children to provide weight and lifestyle advice?

6. What support / information / training do you feel you need to help you encourage patients to acknowledge and address their overweight or obesity?

7. What tools would help you support parents whose children are overweight or obese?

8. How would you rate your confidence in terms of addressing a patient's overweight / obesity healthcare needs? Choose a description and then rank it numerically with one being the lowest and three being the highest for each description.

9. Please describe your positive experiences in this field of healthcare what worked well?

10. Please describe your negative experiences in this field of healthcare what made it a negative experience for you / your patient? 
11. If your personal BMI is outside the healthy range - what strategies do you use to encourage patients to acknowledge their overweight or obesity, and to address their health needs?

12. Have you ever identified safeguarding as an issue related to your patients' overweight or obesity?

a. Do you think it may sometimes be a linked factor?

b. If so, what steps did / would you take in relation to addressing this?

13. How confident do you feel about being able to translate your findings from the growth charts into meaningful information for the parents?

a. lease share any particular successes / challenges you have had with this.

14. What do you think an educational platform should offer in terms of functionality?

a. a place to ask questions

b. case studies

c. videos

d. multiple choice questions

e. test your knowledge sections

f. signposting section

g. brief interventions

h. longer term care options 
i. a way to personalise the learning experience such as an avatar to walk through the learning package with or one to test out new techniques on

j. Please add in anything not mentioned that you would find useful or if you want to choose more than one answer above please list them numerically here (top choice is number 1)

15. How and when do you think you would use a learning platform designed to support you with addressing overweight / obesity in children?
a. one you could use during the patient consultation
b. one for your own learning only
c. Other (please specify) 